\title{
NON-AQUEOUS COLLOID SYSTEMS WIIH SPECIAL REFERENCE TO NITROCELLULOSE
}

BY

\author{
F. SPROXTON, B.Sc., F.I.C.
}

Nearly all nitrocellulose solvents contain the carbonyl group - $\mathrm{CO}$-in the form of a ketone, ester, or acetyl group. There are, however, some exceptions, and ether-alcohol is one of considerable importance, particularly in view of its extensive use during the war in cordite manufacture. Baker ${ }^{1}$ investigated the viscosity of etheralcohol mixtures and concluded that dissociation of (associated) alcohols takes place in such mixtures, but is accompanied by the formation of an ether-alcohol complex, to which the solubility of nitrocellulose is due. Quite recently, Gibson and $\mathrm{McCall}^{2}$ have investigated the effect of variations in the proportions of ether and alcohol on the viscosity of ether-alcohol solutions of nitrocellulose. They find that "the optimum solvent for a nitrocellulose depends on its nitrogen content, and that the higher the nitrogen content, the more ether the optimum solvent requires." This method of expressing the result is, perhaps, open to criticism. The composition of the optimum solvent may vary with the nitrogen content, but it may be directly governed by some other factor which usually varies at the same time as the nitrogen content. The variable factors in nitrocellulose are: (1) the structural complexity of the original cellulose; (2) the complexity of the cellulose after nitration; (3) the percentage of nitryl groups; (4) the percentage of free hydroxyl groups; (5) minor functions such as ketonic or aldehydic groups, and sulphuric acid esters. It is possible that the free hydroxyl groups have a considerable influence on the solubility relations. Dry nitrocellulose absorbs moisture from a damp atmosphere. In fact, Will, ${ }^{3}$ in 1905, came to the conclusion that the sum of nitrogen percentage plus water absorbed was a constant $(14 \cdot 6)$. This conclusion was based on the examination of 65 samples of nitrocellulose. Macdonald's observations on the composition of the spent acids from the displacement process 4 are best explained by assuming slight hydrolysis of the nitrocellulose when the nitrating acids are displaced by water. Now, it has been shown by Renker ${ }^{5}$ that cellulose dried by alcohol retains traces of alcohol even when dried at $130^{\circ} \mathrm{C}$. It is not impossible, therefore, that the free hydroxyl groups in nitrocellulose attract alcohol molecules which would otherwise take part in the formation of the solvent complex, and the variations in the composition of the optimum ether-alcohol solvent for various nitrocelluloses may be due to the differences in the percentage of hydroxyl groups which the nitrocelluloses contain. A simple calculation will show that (1) if Will's generalisation is correct, (2) if the hygroscopicity of nitrocellulose is a measure of its free hydroxyl content, and (3) if the free hydroxyls withdraw alcohol from the ether-alcohol mixture, the results found by Gibson and McCall are qualitatively in agreement with the theory that the solvent action of ether-alcohol is due to the formation of a definite ether-alcohol complex. Baker's theory is not, therefore, definitely disproved by Gibson and McCall's observation. Chandelot, in $1912^{6}$, investigated the interesting fact that 
moist nitrocellulose is more soluble in ether-alcohol than dry. Masson and McCall ${ }^{7}$ have recently published a paper on the viscosity of nitrocellulose in mixtures of acetone and water, showing that the minimum viscosity is obtained not with dry acetone, but with acetone containing a small percentage of water, varying, for instance, from 8 to 10 per cent. for a sample of nitrocellulose containing $12 \cdot 3$ per cent. of nitrogen, and from 6 to 7 per cent. for a sample containing 13 per cent. of nitrogen. Here, also, there is a suggestion that the free hydroxyl groups of the nitrocellulose modify its solubility relations, and that what really passes into solution is nitrocellulose + water molecules attracted by its free hydroxyl groups. In the case of dilute solutions, e.g., where the concentration of nitrocellulose is about $0 \cdot 1$ per cent., in technically pure acetone (not specially dried), a minimum viscosity is not found as the water content increases. There is a continuous rise to a maximum value and then a fall as the solution is converted to a suspensoid sol. If a minimum occurs, it must be at a lower concentration of water than that normally present in technically pure acetone. It seems probable that in these aqueous-acetone solutions of nitrocellulose we have nitrocellulose present in all degrees of state from emulsoid to suspensoid, and this suggests the question whether it is possible, in the same solution, for emulsoid nitrocellulose to act a protecting colloid to suspensoid nitrocellulose. Mardles, Moses and Willstrop ${ }^{8}$ note that water, up to 6 per cent., increases the solvent power of acetone for cellulose acetate, and their paper contains a great deal of interesting information about this cellulose ester. One of their methods of investigation involves the determination of "transition temperature," i.e., the temperature at which a non-solvent for cellulose acetate becomes a solvent. Since there are many transition temperatures, this might be called the Tyndall point. If a solvent is diluted by an indifferent non-solvent, such as petrol, the transition temperature is raised, and by extrapolation a value can be found for the transition temperature of the petrol. This value is artificial, because it is frequently far above the decomposition temperature of cellulose acetate, but it is useful as an index of solvent power. In the case of many binary mixtures, it is found that the curve connecting the composition of the mixture with the transition temperature shows a minimum. Such results are, of course, parallel to those obtained for the viscosity of the nitrocellulose in etheralcohol, and it is interesting to note that the authors find that the minima in the cases of tetrachlorethane and alcohol, butyl formate and alcohol, methyl benzoate and alcohol, approximate very closely to mixtures in simple molecular proportions, which, again, suggests that the solubility is due to the formation of a molecular complex in such mixtures of substances which are themselves non-solvents.

In a contribution to the third British Association Report on Colloid Chemistry, I have mentioned another method of investigating such binary mixtures, which consists in making use of a nitrocellulose of incomplete solubility, and determining the percentage dissolved by various mixtures of the liquids under examination. Alcohol and toluene show a maximum solvent power for the samples of nitrocellulose tested, at a concentration corresponding closely to three molecules of alcohol to one molecule of toluene. The toluene and acetone solubility curve shows no maximum, the solvent power of the mixture decreasing continuously as the percentage of toluene increases. The addition of a constant percentage of petroleum spirit 
to alcohol and toluene does not appear to alter the position of the optimum ratio of alcohol to toluene, but reduces the solvent power throughout the curve. The same method has been applied to investigate the solvent powers of mixtures of alcohol and tetrachlorethane for acetyl cellulose. Almost complete solubility has been obtained in mixtures containing between 20 and 60 per cent. of alcohol. It should be observed that this method is not nearly so sensitive as the viscosity method, and less sensitive than the transition temperature method of studying solvent power, but it has some technical advantages. To sum up, the solvent power for cellulose esters can be studied by the following methods:-

(1) Viscosity determinations, the assumption being that that solvent is the best which gives solutions of lowest viscosity.

(2) Determination of transition temperature, i.e., the temperature at which a liquid or mixture of liquids becomes a solvent.

(3) Determination of the amount of an indifferent diluent which may be added to a solution before precipitation takes place.

(4) Determination of the amount dissolved from a sample of cellulose ester of incomplete solubility.

It would be premature to say whether these four methods all lead to the same result, but I have not found any contradictory results up to the present.

Duclaux and Wollman, ${ }^{\circ}$ by the fractional precipitation of an acetone-solution of nitrocellulose by means of water or aqueous acetone, have separated it into fractions of widely different viscosities, although of constant nitrogen content. Since the treatment is equivalent to treating nitrocellulose with aqueous acetone of varying water content, presumably the latter treatment would also fractionate nitrocellulose in this way, and it may be that method (4), given above, has this effect. With regard to the actual peptisation of nitrocellulose, there is little to add to Bancroft's report in the second British Association Report. Masson and McCall, ${ }^{7}$ commenting on the variations of viscosity with time in the case of solutions of nitrocellulose in ether-alcohol and in acetone, remark that two processes appear to be at work: (1) the absorption of solvent by nitrocellulose, accompanied by dispersion of the resulting gel; (2) a change in the already dispersed gel, perhaps in the state of aggregation, not necessariy accompanied by alteration in percentage composition. I have occasionally noticed evidence of the two phenomena mentioned in (1) when preparing nitrocellulose solutions. If, for instance, celloidin (a special form of nitrocellulose in small cubes) is covered with a mixture of 80 per cent. alcohol and 20 per cent. acetone and allowed to stand, after a few days one sometimes notices the formation of two boundaries. The lower one, which is separated from the core of still unswollen nitrocellulose in the centre of the cubes, is usually undulating (corresponding to the original surface of the solid celloidin), and is sharply marked by a difference in refractive index from the supernatant liquid. A few millimetres above this will be seen a horizontal layer, often marked by a layer of fine dust which has fallen through the solvent and been stopped by the slowly-diffusing viscous solution. The lower layer is evidently the boundary of the swollen gel, the upper layer the limit of the dispersion process. The fact that the upper layer is horizontal shows that the viscosity of this phase is low enough to allow relative motion in the liquid. 
The colloid chemistry of the manufacture of solid celluloid is complicated and difficult. Contrary to a somewhat widespread belief, at no stage of the manufacture do we deal with a sol, if we except a momentary formation at the surface of the fibrous nitrocellulose when the liquid solvent first comes into contact with it. The amount of liquid solvent employed is less than the nitrocellulose would take up in its preliminary swelling if soaked in the solvent As a matter of interest the volume of the gel formed under these conditions depends on the concentration of the camphor solution. For example. samples of the same nitrocellulose soaked in alcoholic solutions of camphor of 20 per cent., 30 per cent., and 40 per cent. concentration gave gels in which the specific volume of the nitrocellulose was $4 \cdot 4,3 \cdot 8$, and $3 \cdot 5$ respectively.

Since the product of preliminary swelling is a gel, it follows that the whole of the manufacture of solid celluloid from nitrocellulose deals with the gradual transformation of a gel to a solid. This transformation is accomplished by loss of solvent, which involves loss in weight and in volume, while undoubtedly the mechanical properties of the finished material are influenced also by the amount and character of the manipulation it receives in the plastic state. I have given in the British Association Report some information about the ratio loss of weight/loss of volume in the seasoning process. Since the range of density of all possible solutions of alcohol and camphor at ordinary temperatures is very small, it is interesting to find that the value of the ratio falls within this range. In the seasoning process, the loss of solvent is rapid at first, but a skin of harder and denser celluloid forms on the surface, and seasoning becomes progressively slower as this skin increases in thickness. With moderately thick sheets (e.g., $1 \mathrm{~cm}$. and over) even after the loss of weight has become inappreciable, the centre of the celluloid is softer than the outside, and this fact must be taken account of in any investigation of elasticity, since the usual mathematical theory assumes homogeneity of the material. If a rectangular prism of moderately thick seasoned celluloid is sliced down the middle, each piece will assume a curve with the new surface concave, owing to the escape of solvent and consequent shrinkage. A cube of celluloid of 3 -in. side made many years ago, and now in use as a paper-weight, shows a lens-shaped bubble inside where the soft core has gradually shrunk away from the hard outer shell. The final material is usually regarded as a solid solution of camphor and nitrocellulose, but the traces of alcohol which are retained probably form an integral part of the system. The reasons for the plasticity of celluloid when warm (one "of its most valuable properties) lead to interesting speculations. Nitrocellulose has no melting point, since it decomposes before this point is reached. Camphor melts at $182 \cdot 5^{\circ} \mathrm{C}$. Celluloid becomes appreciably plastic at $80^{\circ} \mathrm{C}$. Therefore the melting point, if the term may be allowed, of the mixture is greatly below that of either constituent. The traces of alcohol present no doubt have a considerable influence in reducing the melting point further than would be the case with camphor and nitrocellulose alone. It may be noted in passing that camphor forms liquid mixtures with several solid substances at ordinary temperatures, and possibly this is one of the properties which make camphor adaptable for celluloid manufacture. Most of the substances which have from time to time been used as camphor substitutes have had much lower melting points than camphor, but the celluloid made from them does not usually become 
plastic at a lower temperature on that account. It would appear, therefore, that the plasticity of warm celluloid is determined not by the melting point of the solid solvent, but by the properties of the complex solid solution formed by nitrocellulose, solid solvent, and liquid solvent. When experimenting with unusual materials, one sometimes encounters limits to the mutual solubility, and the phenomenon occurs known in the industry as "exudation." Mardles, Moses, and Willstrop ${ }^{8}$ mention occasional cases of crystallisation on acetyl cellulose films. This separation of the solid solvent no doubt depends on its solubility in the liquid solvent and in the nitrocellulose and it would be an interesting phase rule study, although of great experimental difficulty. I have not seen it occur with camphor, probably because of (1) the high mutual solubility of camphor, nitrocellulose, and alcohol; (2) the high vapour pressure of camphor, at ordinary temperatures, which would cause the camphor to volatilise immediately. Probably it could be tracked in the case of camphor by searching for a break in the seasoning process, using a sample of unusually high camphor content.

\section{REFERENCES}

1 Baker, Trans., 1913, 653.

2 Gibson and McCall, J.S.C.I., 1920, 39, 172T-176T.

s Will, Mitt. Centralst. f. Wiss. Tech. Unters, Berlin, 1904, No. 4, 1-33.

4 Macdonald, J.S.C.I., 1911, 30, 251.

5 Renker, "Ueber Bestimmungsmethoden der Cellulose" (Berlin University, 1909 ; Analyst, 1910, 35, 71). . .

6 Chandelon, Bull. Soc. Chim. Belg., 1912, 26, 495-506.

7 Masson and McCall, Trans., 1920, 117, 819-823.

8 Mardles, Moses and Willstrop; Advisory Committee for Aeronautics; Reports and Memoranda, No. 568.

9 Duclaux and Wollman, Bull. Soc. Chim., 1920, 27, 414-420. 